\title{
RECURRENT DUST FORMATION BY WOLF-RAYET STARS
}

\author{
P.M. WILLIAMS ${ }^{1}$, K.A. VAN DER HUCHT ${ }^{2}$, P.S. THÉ ${ }^{3}$, \\ P. BOUCHET ${ }^{4}$ and G. ROBERTS ${ }^{5}$ \\ 1 Royal Observatory, Blackford Hill, Edinburgh EH9 3HJ, United Kingdom \\ 2 SRON Laboratory for Space Research, \\ Sorbonnelaan 2, 3584 CA Utrecht, The Netherlands \\ 3 Astronomical Institute 'Anton Pannekoek', \\ Roetersstraat 15, 1018 WB Amsterdam, The Netherlands \\ 4 European Southern Observatory, Karl-Schwarzschild-Strasse 2, \\ D 8046 Garching bei München, F.R. Germany \\ 5 S.A. Astronomical Observatory, P.O. Box 9, Observatory 7935, \\ South Africa
}

\begin{abstract}
A number of Wolf-Rayet stars show variations of up to a factor of ten in their infrared emission on timescales of months to years while their photospheric luminosities remain unchanged. This can be interpreted in terms of variation in the rates at which dust grains form in their stellar winds. Our data show variable circumstellar dust emission from WR 70 (HD 137603), with an episode of enhanced dust formation in early 1989, and fading of emission by dust formed around WR 48a (in 1979) and WR 19 some time before our first observation in 1988. We consider their relation to WR 140 (HD 193793), which forms dust in its wind for a few months at intervals of 7.94 years.
\end{abstract}

\section{WR 48a: a decade of slowly cooling dust}

This is a visually faint star discovered in the infrared by Danks et al. (1983) as it rose to maximum in 1979. We have been monitoring its steady fading since 1982 and present in Fig 1 a $2.2 \mu \mathrm{m}(\mathrm{K})$ light curve showing fading at a rate of about $0^{\mathrm{m}} \cdot 26 /$ year. The fading has been slower at longer wavelengths, e.g. $0^{\mathrm{m}} \cdot 11$ /year at $10 \mu \mathrm{m}$ and $0 \cdot 22 /$ year at $3.8 \mu \mathrm{m}$, indicating that the dust is cooling. At shorter wavelengths, e.g. $1.25 \mu \mathrm{m}$, the fading was initially faster, as we would expect from cooling dust, but then slowed down and stopped (in 1989) as the dust emission became comparable with that from the stellar photosphere and wind. 


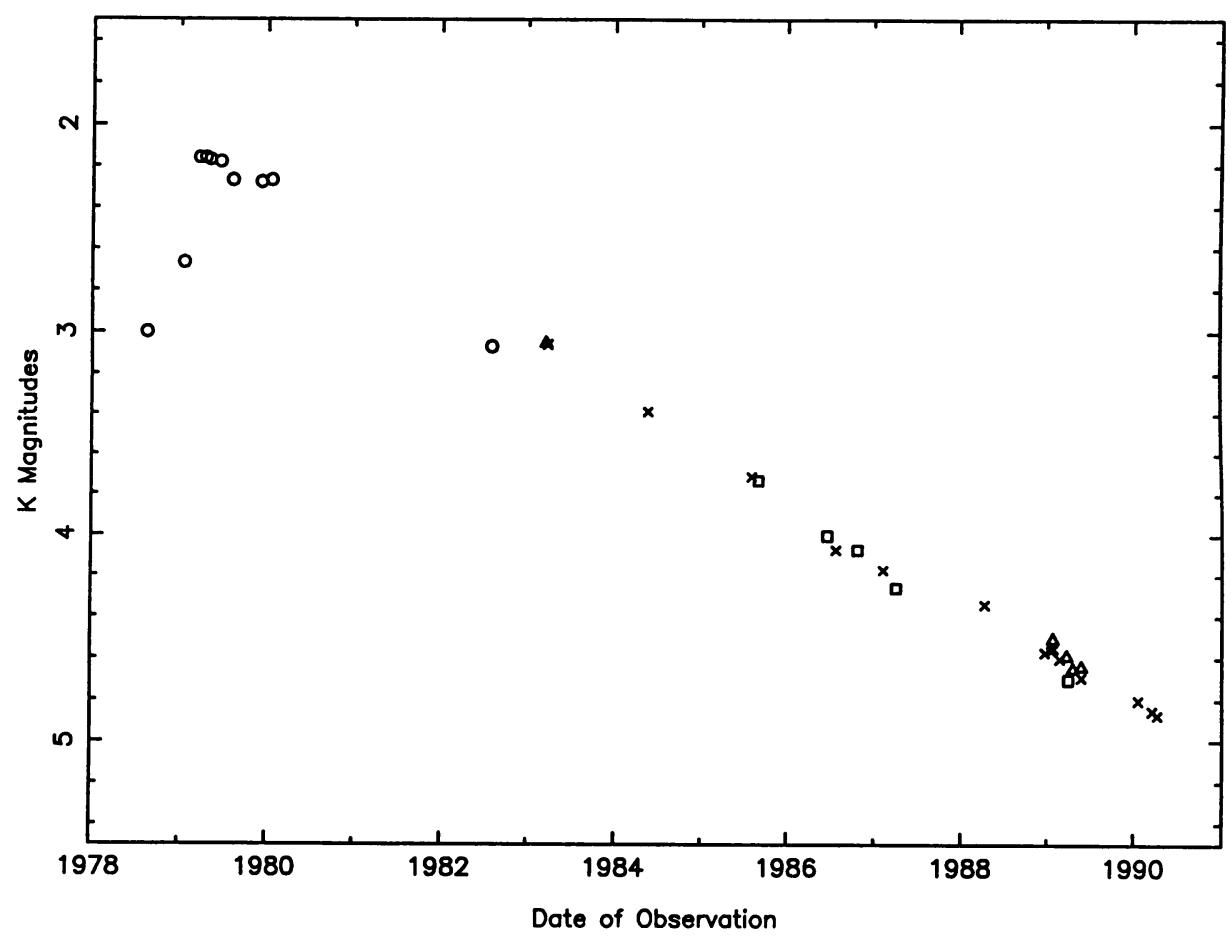

Fig. 1. Infrared light curve of WR 48 a based on observations made at ESO $(\times)$, the SAAO $(\triangle)$ and the AAT (口) and those published by Danks et al. (०).

In order to model the evolution of the cooling dust shell, as was done for that of WR 140 (Williams et al. 1990), we need to know the 'baseline' infrared energy distribution of WR 48a before the dust condensation occurred. Initially, we assume this to be free-free radiation from the stellar wind alone. Modelling the infrared spectra yields the dust temperatures at the times of observation and, from consideration of the thermal equilibrium of the grains in the stellar radiation field, the distances of the dust shell from the central star. We need to know the luminosity of WR 48 a to determine the expansion velocity in this way, but the velocities derived $(\approx 30 \mathrm{~km} / \mathrm{s})$ for reasonable estimates are so much lower than plausible values of the (also unknown) velocity of WR 48a's stellar wind that we have to question our initial assumption about the pre-outburst spectrum of WR 48a. If this showed emission by dust formed in an earlier event which had cooled by expansion, then the dust temperatures derived from the 1979-1990 observations cover a larger range, yielding a higher value for the expansion velocity. This suggests that, although only one has been observed, dust formation episodes from WR 48a may be recurrent - as is the case with WR 140. Besides continued monitoring of the circumstellar dust, we need to learn more about the star in WR 48a, particularly whether or not it is a binary like WR 140, whose dust formation was linked to its orbital motion by Williams et al. (1990). 


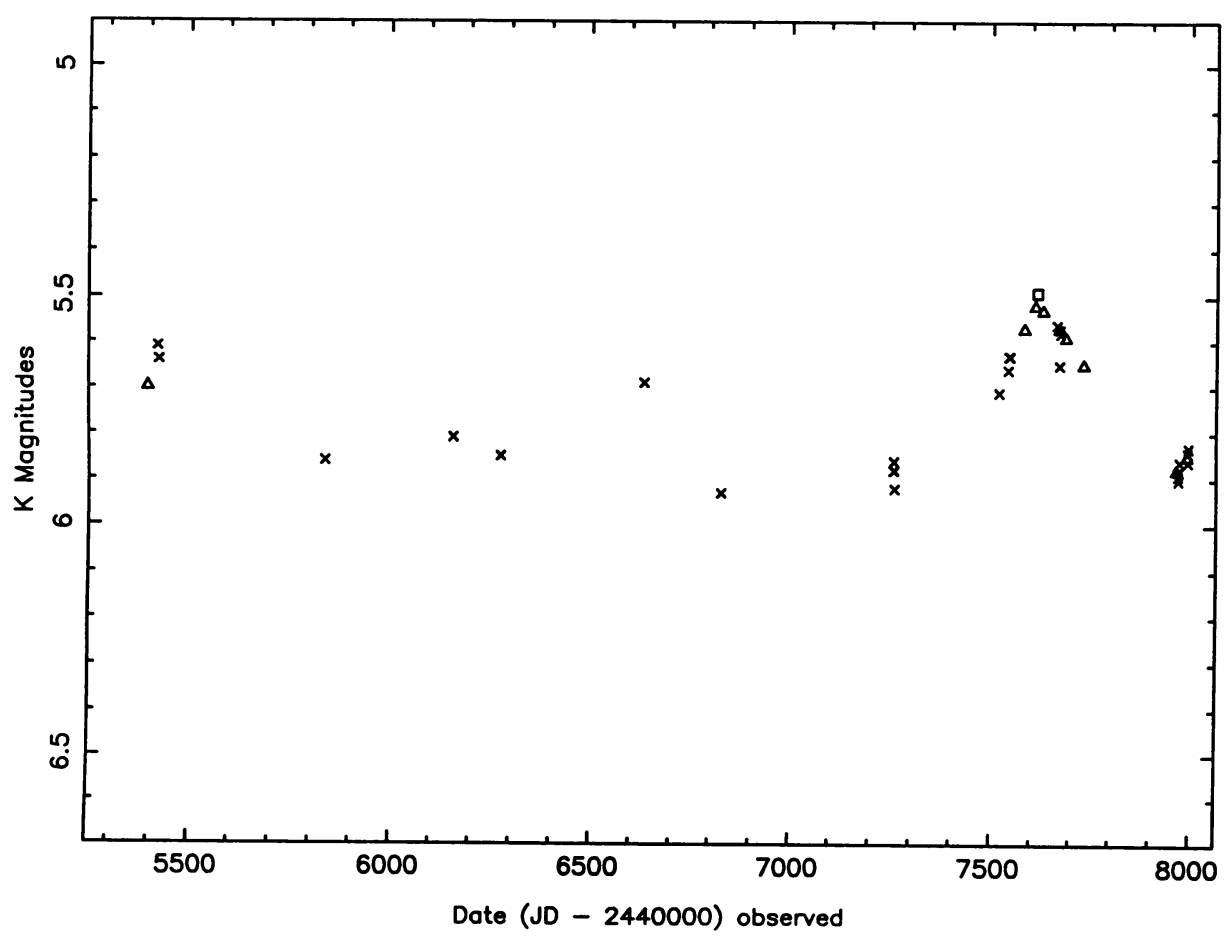

Fig. 2. Infrared light curve of WR 70 (symbols as in Fig 1.).

\section{WR 70: a binary with variable dust formation}

The WC8+B0 binary system WR 70 (= HD 137603) differs from WR 48a and WR 140 in showing variations on a shorter timescale $(\approx 1-2$ years $)$ and of significantly lower amplitude $\left(\approx 0 \mathrm{~m}_{5}\right.$ in $\left.\mathrm{K}\right)$. Like the other two cases, the amplitude increases with wavelength $(\approx 0 \cdot 6$ in $\mathrm{M}$ but only $\approx 0 \cdot 3$ in $\mathrm{H}$ ), indicating that it is the amount of circumstellar dust that is varying rather than the underlying star. The (K-L) and (K-[3.8]) colours get progressively redder as the flux rises to and falls from the well-defined maximum around JD 2447650 (1980 March). This observation that the dust is hotter during the rise indicates that the rise is caused by an increase in the amount of dust condensing in the wind at the inner edge of the dust shell for 3-6 months and the fall by the carrying away of this extra dust in the wind after this dust formation ceases. More intensive observations are needed to characterize such events, confirm their recurrence and establish the timescale of the variations.

We recall that, like WR 140 , WR 70 is a binary so that the relation of its dust formation episodes to its orbital motion will be of particular interest. The WR 70 system is bright enough in the visible and even UV for detailed study. 


\section{WR 19: fading dust emission from a WC4 star}

Our first observation of WR 19 (LS 3) in 1988 April showed it to have infrared fluxes characteristic of a circumstellar dust shell. Subsequent observations have shown a steady fading of this emission until 1990, when only the free-free emission from the stellar wind was apparent. Like WR 48a, this is a relatively faint star in a difficult field and so will not be easy to study. On the other hand, it is potentially most important, showing that dust formation can occur around stars as early as WC4. As searches for circumstellar dust emission have hitherto been concentrated on stars of WC8 and later, it is likely that dust formation in the winds of Wolf-Rayet stars may be more common than previously thought and that only continuous dust formation is restricted to those of the later spectral subtypes.

\section{ACKNOWLEDGEMENTS}

We are grateful to the following for contributing observations: David Allen, Brian Carter, Michael Feast, R. de Jong, Dave Laney, A. Leene, Fred Marang, Pieter Mulder, Frank Spaan, R. Vega, Patricia Whitelock, Hartmut Winkler and Dolf de Winter.

\section{REFERENCES}

Danks, A.C., Dennefeld, M., Wamsteker, W. and Shaver, P.A., 1983. Astr. Astrophys. 118, 301.

Williams, P.M., van der Hucht, K.A., Pollock, A.M.T., Florkowski, D.R., van der Woerd, H. and Wamsteker, W.M., 1990. M.N.R.A.S. 243, 662. 